\title{
Fuzzy TOPSIS Multi-Criteria Decision Analysis Applied to Karun Reservoirs System
}

\author{
Amin Afshar • Miguel A. Mariño • Motahareh Saadatpour • \\ Abbas Afshar
}

Received: 23 June 2009 / Accepted: 20 September 2010 /

Published online: 16 October 2010

(C) The Author(s) 2010. This article is published with open access at Springerlink.com

\begin{abstract}
Water resource systems, with an abundance of project purposes and resource values, are subject to conflicting policy, planning, and management decisions. Multi-criteria decision making methods (MCDM) provide a framework to help water managers identify critical issues, attach relative priorities to those issues, select best compromise alternatives, and facilitate communication to gain general acceptance. This paper addresses a method that incorporates several system factors/components within a general framework for providing a holistic analysis of the problems and comprehensive evaluation of the related mitigation/adaptation measures and policy responses. The method accounts for uncertainties in both the quantification and importance of objectives in the ranking process. The proposed fuzzy multi-criteria decision making process uses the well known Technique for Order Preference by Similarity of Ideal Solution (TOPSIS) method in both deterministic and uncertain environments. The performance of the proposed approach to a real water resource management problem in Iran is illustrated. Results show that the model may be used in a large-scale multi-level assessment process. Ranks of the alternatives are presented using deterministic and fuzzy based models.
\end{abstract}

\footnotetext{
A. Afshar $(\varangle)$

Department of Civil and Environmental Engineering, University of California, Davis, Davis, CA, USA

e-mail: afshar@ucdavis.edu
}

\section{A. Mariño}

Department of Civil and Environmental Engineering and Department of Land, Air and Water Resources, University of California, Davis, Davis, CA, USA e-mail: MAMarino@ucdavis.edu

\section{Saadatpour}

Department of Civil and Environmental Engineering, Iran University of Science and Technology, Tehran, Iran

\section{A. Afshar}

Department of Civil and Environmental Engineering, and Enviro-Hydroinformatic Center of Excellence, Iran University of Science and Technology (IUST), Tehran, Iran 
Keywords TOPSIS • MCDM • Fuzzy • Water resources • Uncertainty • Karun river basin

\section{Introduction}

Water resource systems, with very diverse purposes and resource values, are subject to conflicts over water management decisions. Most often these conflicts arise because water resource systems are managed to optimize conflicting benefits for water supply, hydropower, recreation, and/or flood control. Although these benefits are usually quantified in monetary values, natural and environmental resources are often difficult, if not impossible, to be quantified in economic terms (Flug et al. 2000). In optimization approaches to systems analysis it is most convenient to describe all objectives in commensurable units. In spite of extensive research efforts in this area, to date this remains difficult and controversial. This difficulty is more pronounced for natural and cultural objectives, which are often evaluated in qualitative terms only.

Management of water resource projects is highly complicated partially due to their multi-period, multilayer, and multi-objective features. For a given goal, many alternative options may exist with different levels of satisfaction for the related decision makers and stakeholders. It is, if not impossible, very difficult to clearly identify the "best" among them (Goicoechea et al. 1982). Optimal ranking of water projects with conflicting objectives (environmental, physical, socio-economic, and health) can be very complex issue and challenging.

Today Multi-Criteria Aid (MCA), as an established methodology, has received particular attention in water resource management, partly because water policy is seldom guided by a single objective. MCA can be defined as a decision model which contains (Figueira et al. 2005a):

- A set of decision options to be ranked by the decision maker

- A set of criteria, typically measured in different units

- A set of performance measures, which scores and/or ranks each decision option against each criterion.

There is a need for reliable methodology to select and rank water projects and alternatives according to different objectives. During last decades, techniques dealing with complex problems being characterized by mixtures of quantitative and qualitative objectives, have received considerable attentions. These techniques establish preferences between alternatives to an explicit set of objectives and measurable criteria (Mourits and Oude Lansink 2006; Figueira et al. 2005a).

Multi-criteria decision analysis (MCDA) methods provide a framework to help water managers identify critical issues, assign relative priorities to those issues, select compromise alternatives, and facilitate communication to gain general acceptance between stakeholders (Hajkowicz and Collins 2007).

The Technique for Order Preference by Similarity of Ideal Solution (TOPSIS), with no articulation of preference information, was initially proposed for solving multiple attribute decision making problems (Hwang and Yoon 1981). With a given reference point, MCDM problems can then be solved by locating alternatives or decisions which are closest to a reference and/or ideal point. 
The aim of this research is to address a technique incorporating multi-level analysis to determine the actual state of the water related environment from joint ecological and socioeconomic perspectives. Based on this technique, a holistic analysis and comprehensive evaluation of the project and/or related mitigation/adaptation measures and policy responses in a large-scale water resource development project is accomplished considering several system factors/components within a general framework. The proposed multi-criteria decision making approach includes not only those factors within the water system itself but also other socioeconomic, ecological, environmental, legislative, and political issues due to their direct or indirect relations to water management. The method is able to account for uncertainties in both the quantification and importance of objectives and/or criteria in the ranking. The proposed approach has been applied to the Karun river basin in Iran where the ministry of energy places high priority on economic return and energy development, environmental interest groups prefer preserving the natural ecosystem, and native people are more concerned about their living style and local culture, which is closely related to habitat protection and community stability.

The proposed method starts with evaluating the actual state of the system as: (1) Basic indicators are defined, (2) Maximum and minimum values for the basic indicators are determined, (3) basic indicators are grouped according to defined secondlevel composite indicators, and (5) second-level composite indicators are grouped according to the composite ecological indicators and the composite socioeconomic indicator. Then the second-level and the third-level composite distance functions are calculated from the first and second-level distance functions, respectively. The composite distance from the reference point (ideal solution) is then minimized to locate the most desirable solution, as discussed in detail in the next sections (UNEP 1987).

\section{MCDM Approaches in Water Resources Alternative Ranking}

Multi-criteria decision making (MCDM) refers to the process of screening, prioritizing, ranking, or selecting a set of alternatives under usually independent, incommensurate, and/or conflicting criteria (Larichev and Moshkovich 1995; Raju et al. 2000).

A wide variety of MCDM models has been developed to help a decision maker (DM) select from, at least partly conflicting, finite sets of alternatives according to two or more criteria. An MCDA problem consists of a set of alternatives and/or options, $A=\left\{A_{1}, A_{2}, \ldots, A_{j}, \ldots, A_{n}\right\}$, and a set of criteria $C=$ $\left\{C_{1}, C_{2}, \ldots, C_{i}, \ldots, C_{m}\right\}$. The consequence on criterion $i$ of alternative $A_{j}$ is expressed as $x_{i j}$. The importance of each criterion is usually given in a one dimensional weights vector $\boldsymbol{W}$, where $w_{i}$ denotes the weight assigned to the ith criterion. Although not universal, Hajkowicz and Collins (2007) outline the most common stages in a MCDM process.

As outlined by Hajkowicz and Collins (2007), MCDM techniques may be classified as (1) multi-criteria value functions (Lai et al. 2008), (2) outranking approaches (Figueira et al. 2005b), (3) goal or reference point method (Abrishamchi et al. 2005), (5) pairwise comparisons (Saaty 2004), and (6) tailored methods (Hyde et al. 2004). 
Goal programming (GP) and compromise programming (CP) are two major methods which fall into goal or reference point method. The underlying principle of GP is to eliminate alternatives until a satisfactory level of performance for each criterion is achieved. The $\mathrm{CP}$ is an extension of GP in which some measure of distance is used to come as close to the ideal solution as possible. These approaches identify the reference levels as ideal and anti-ideal values for the criteria. Then the closest decision options to the ideal and furthest from the anti-ideal are identified. Where no ideal or anti-ideal is easily defined, the minimum and maximum criterion values may be used. Compromise programming and TOPSIS are among the most common techniques of this type.

In an extensive critical survey of 94 papers in water resource applications of conventional MCA methods, Hajkowicz and Collins (2007) found out that, disregarding the specific methods within the category, distance to ideal point approach has been employed more frequently (Table 1). TOPSIS was reported as the least frequently used method within the category. Although there is a controversy in accepting fuzzy set analysis as a MCDM method by itself, it has been ranked as the second in this survey. In fact, it combines different MCDM approaches with fuzzy set concepts to tackle some of the possibilistic uncertainties in the values and/or weights of the criteria (Hajkowicz and Collins 2007).

The applications cited by Hajkowicz and Collins (2007), names some more important articles in different areas of water management. Without downgrading other valuable researches, few related works belonging to the third class (goal or reference point method including TOPSIS) are cited as follows. Gershon et al. (1982) dealt with multi-objective optimization of river basin planning. MCDM approach was employed to select a best wastewater management alternative by Tecle et al. (1988). Simonovic (1989) formulated a MCDM model for ranking national water master plans in Canada. Flug et al. (2000) applied the weighted sum method to evaluate nine discrete flow release alternatives in managing the Colorado River system below Glen Canyon Dam. Kheireldin and Fahmy (2001) applied Compromise Programming to evaluate Egypt's long term water strategies under several social, economic, and environmental factors. They concluded that while this MCDA approach provided an efficient and systematic way of presenting tradeoffs among policy choices, it also provided transparency for negotiation among different stakeholders. Some other major contributions are on ranking groundwater management alternatives (Duckstein et al. 1994), design of long-term water supply in Southern France (Netto

Table 1 Water resource applications of conventional MCA methods

\begin{tabular}{lll}
\hline Category & MCA method & Number of application \\
\hline Distance to ideal point & Various methods (CP, TOPSIS, ...) & 24 \\
Fuzzy set analysis & Fuzzy set analysis & 22 \\
Pair wise comparisons & Analytic Hierarchy Process & 15 \\
Outranking methods & ELECTRE I, II, III, IV and TRI & 15 \\
Outranking methods & PROMETHEE I, II, V & 12 \\
Multi-criteria value function & Multi attribute utility theory & 8 \\
\hline
\end{tabular}


et al. 1996), conflict resolution in water resource planning (Cai et al. 2004), and urban water supply planning (Abrishamchi et al. 2005).

Most real-world water resource problems are characterized by various degrees of uncertainty. Ashtian et al. (2009) presented an interval-valued fuzzy TOPSIS method to solve MCDM problems in which the weights of criteria are unequal. Inexact and interval-valued fuzzy approach has been proposed and applied to solid waste management problems in uncertain environments (Tan et al. 2009; Cai et al. 2009). To directly cope with linguistic models of human interpretation of environmental systems, Borri et al. (1998) introduced a fuzzy rule-based methodology for environmental evaluation. Gonzalez et al. (2002) utilized fuzzy logic to relax the need for in-depth environmental knowledge and extremely accurate data to conduct the assessment study. Very often crisp data are inadequate to model real life situations. Triantphyllou and Lin (1996) employed fuzzy arithmetic operations to develop a fuzzy version of TOPSIS method which leads to a fuzzy relative closeness for each alternative. Morón et al. (2009) presented a fuzzy EIA model which incorporates both qualitative and quantitative information, using fuzzy set theory. They also presented a software program which implements this fuzzy methodology for environmental impact assessments. Liu et al. (2009) utilized fuzzy logic as a decision support approach for environmental impact assessment. The evaluation knowledge represented by "if-then" fuzzy rules is used to measure the significance of environmental impacts. Chen (2000) described the rating of each alternative and the weight of each criterion by linguistic terms which were expressed in triangular fuzzy numbers. A vertex method was proposed to calculate the distance between two triangular fuzzy numbers. According to the concept of the TOPSIS, a closeness coefficient was defined to determine the ranking order of all alternatives by calculating the distances to both the fuzzy positive-ideal solution (FPIS) and fuzzy negative-ideal solution (FNIS) simultaneously. Peche and Rodríguez (2009) proposed an approach based on fuzzy logic to carry out the environmental impact assessment (EIA) of activities and projects.

The choice of an appropriate method depends on the characteristics of the system being considered, on availability of data, and on the objectives and constraints specified (Barros et al. 2003).

\section{Problem Formulation with UNEP Method; TOPSIS Approach}

The TOPSIS method was initiated for solving a multiple attribute decision making problem with no articulation of preference information (Hwang and Yoon 1981). TOPSIS technique is based on the concept that the ideal alternative has the best level for all attributes considered, whereas the negative ideal is the one with all the worst attribute values. A TOPSIS solution is defined as the alternative which is simultaneously farthest from the negative-ideal and closest to the ideal alternative (Chu 2002).

Due to incommensurability among the criteria, one has to normalize the distance family to minimize and practically reduce the effects of the incommensurability. To obtain a compromise solution, the normalized distance family to the ideal solution 
may be used. The problem then becomes how to solve the following auxiliary problem which defines the distance between two points, $f(x)$ and $f\left(x^{*}\right)$ (the reference/ideal point), in $k$ dimensional space as:

$$
\min _{x \in X} d_{p}=\left(\sum_{t=1}^{k}\left(f\left(x_{t}\right)-f\left(x_{t}^{*}\right)\right)^{p}\right)^{1 / p}
$$

or

$$
\min _{x \in X} d_{p}=\left(\sum_{t=1}^{k}\left(\frac{f\left(x_{t}\right)-f\left(x_{t}^{*}\right)}{f\left(x_{t}^{*}\right)}\right)^{p}\right)^{1 / p}
$$

in which $p$ addresses the importance of deviation from the ideal situation, and $x^{*}$ is the ideal solution. The value of $p$ reflects the way of achieving a compromise by minimizing the weighted sum of the deviations of criteria from their respective reference points.

The United Nation Environmental Program (UNEP) has proposed a methodology and guideline for assessing water resource development projects. The methodology employs a TOPSIS type approach. The first section of the procedure refers to the evaluation of the actual state of the system. It is performed by defining the basic indicators, determining the maximum and minimum values for the basic indicators, grouping the basic indicators according to defined second-level composite indicators, and grouping second-level composite indicators according to the composite ecological indicators and the composite socioeconomic indicator.

Let $A$ be the set (discrete or continuous) of management options, and $A_{j}$ be the $j$ th element of this set. Next, the relationship $Z_{j}\left(x_{i}\right)$ between the options $A_{j}$ and the resulting values of the basic indicators $\left(x_{i}\right)$ should be estimated. This step can be performed with different degrees of sophistication. Clearly, a detailed analysis would involve use of several prediction models both for indicators in the ecology and socioeconomics environments. Since units of basic indicators are different, the further trade off analysis requires that the actual values be normalized, using the maximum and minimum $Z^{i}\left(\max Z^{i}\right.$ and $\min Z^{i}$, respectively) (Eq. 3a and Eq. 3b) to produce index function of $S_{j}\left(x_{i}\right)$.

$$
\begin{aligned}
& S_{j}\left(x_{i}\right)=\frac{\max Z^{i}-Z_{j}\left(x_{i}\right)}{\max Z^{i}-\min Z^{i}} \\
& S_{j}\left(x_{i}\right)=\frac{Z_{j}\left(x_{i}\right)-\min Z^{i}}{\max Z^{i}-\min Z^{i}}
\end{aligned}
$$

Where $\max Z^{i}=\max _{j} Z_{j}\left(x_{i}\right)$ and $\min Z^{i}=\min _{j} Z_{j}\left(x_{i}\right)$.

Next, second-level composite distance functions are calculated as:

$$
L_{j}^{(2)}(x)=\left[\sum_{i=1}^{n_{i}} \alpha_{i j} . S_{j}\left(x_{i}\right)^{P_{i}}\right]^{1 / p_{i}}
$$

Where, $n_{i^{\prime}}$ is the number of basic indicators in group $i^{\prime}, \alpha_{i i^{\prime}}$ are weights expressing the relative importance of basic indicator $i$ in group $i^{\prime}$, and $P_{i^{\prime}}$ is the balancing factor among indicators in group $i^{\prime}$. The weighting factors $\alpha_{i i^{\prime}}$ and $P_{i^{\prime}}$ should be 
assessed. The weighting scheme is assumed to be independent of the various options. Further, third-level composite distance functions are calculated from the secondlevel distance function as:

$$
L_{j}^{(3)}(x)=\left[\sum_{i=1}^{m_{k}} \alpha_{i^{\prime} j} \cdot L_{i^{\prime} j}^{(2)}\left(x_{k}\right)^{P_{k}}\right]^{1 / p_{k}}
$$

Where $m_{k}$ and $P_{k}$ are the number of elements and balancing factor among indicators in group $k$ (ecology and socio-economy in second-level). Finally, the system composite distance function can be calculated as Eq. 6 .

$$
L_{j}(x)=\left[\alpha_{1} \cdot L_{1}^{(3)}(x)^{2}+\alpha_{2} \cdot L_{2}^{(3)}(x)^{2}\right]^{1 / 2}
$$

Equation 6 shows that the systems composite indicator, $L$, is a function of option $A_{j}$, basic indicator, relationship $Z(x)$, and a weighting scheme $\alpha$ and $p$. The next step of the procedure selects the most desirable option from the available ones. The option with the closest state to the ideal state is selected as the best option. Since the system composite index $L$ measures the distance from the ideal state, the best option will correspond to the minimum value of $L(x)$.

\section{Fuzzy-Based Approach to TOPSIS MCDM (UNEP Method)}

Classical TOPSIS methods assume that the ratings of alternatives and the weights of criteria are crisp numbers. Increasingly, this is recognized as unrealistic. Generally, uncertainties arise from unquantifiable, incomplete, unobtainable information, and/or partial ignorance. A variable's value can be uncertain, both, if it is singlevalued, deterministic, and constant with imperfectly known value, or if its value is constantly fluctuating with a random pattern (Lund 1991). Beside the probabilistic analysis, many other forms of analysis have been proposed to integrate uncertainty into decision analysis. These include fuzzy sets, analytic hierarchy process (AHP), sensitivity analysis, "robust" analysis, etc. (Lund 2008). Since classical TOPSIS method cannot handle problems with imprecise information, the representation and interpretation of "uncertainty" and human-related subjective preference may be helpful. Fuzzy set theory (Zimmermann 1996) seems to have been the most commonly used method in accounting for such vague and imprecise information (Hajkowicz and Collins 2007).

Uncertainty in water resource management problems can be attributed to the random or fuzzy character of the information available. Fuzziness is non-statistical in nature and refers to the absence of sharp boundaries in the information. This imprecision is generally due to the inclusion of human judgments and preferences in a problem formulation. Fuzzy set theory has extensively been applied in MCDM processes (Zhou et al. 1999; Fu 2008).

Fuzzy multi- criteria decision making model (FMCDM) utilizes linguistic variables and fuzzy numbers to aggregate the decision makers' subjective assessment about criteria weightings and appropriateness of alternative candidate versus selection criteria to obtain the final scores-fuzzy appropriateness indices ( Fu 2008). 
To consider fuzzy, as opposed to crisp, values in $D$ (performance matrix) and $W$ are presented as Eqs. 7 and 8.

$$
\begin{gathered}
\tilde{D}=\left[\tilde{x}_{i j}\right] \\
\tilde{W}=\left(\tilde{w}_{i}\right)
\end{gathered}
$$

Where $\tilde{x}_{i j}$ represents the fuzzy rating of alternative $A_{j}$ with respect to criterion $C_{i}$, and $\tilde{w}_{i}$ represents the fuzzy weight for criterion $C_{i}$.

In the absence of a reliable probability distribution function, an intuitively easy, effective, and commonly used approach to accounting for uncertainty about the value of an unknown parameter is a triangular fuzzy number $\tilde{x}_{i j}=\left(a_{i j}, b_{i j}, c_{i j}\right)$. In applications it is often convenient to work with triangular and/or trapezoidal fuzzy numbers because of their computational simplicity, and they are useful in promoting representation, and information processing in a fuzzy environment (Ertuğrul and Güneş 2007; Ertuğrul and Karakaşoğlu 2008).

The fuzzy performance matrix is formed by arraying columns of alternatives with rows of criteria as shown below.

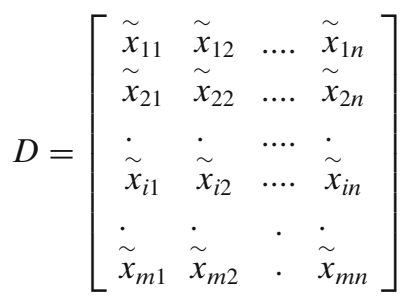

Where $\tilde{x}_{i j}$ represents the performance of alternative $A_{j}$ with respect to attribute $C_{i}$. The performance matrix should be normalized to convert the values of alternatives into a common dimensionless unit for comparison. The normalized performance $\tilde{x}_{i j}$ can be obtained using the following transformation formulae for benefit criteria and cost criteria, respectively.

$$
\begin{gathered}
\tilde{r_{i j}}=\left(\frac{a_{i j}}{c_{i}^{+}}, \frac{b_{i j}}{c_{i}^{+}}, \frac{c_{i j}}{c_{i}^{+}}\right) \\
\tilde{r_{i j}}=\left(\frac{a_{i}^{-}}{c_{i j}}, \frac{a_{i}^{-}}{b_{i j}}, \frac{a_{i}^{-}}{a_{i j}}\right)
\end{gathered}
$$

Where $c_{i}^{+}=\max _{j} c_{i j}, a_{i}^{-}=\min _{j} a_{i j}$, and $\tilde{r_{i j}}$ represents the normalized performance of $A_{j}$ with respect to attribute $C_{i}$. The matrix form of $\tilde{r_{i j}}$ is given as follows:

$$
\tilde{r}=\left[\tilde{r_{i j}}\right]
$$

By multiply the performance matrix $\tilde{r}$ by its associated weights $\tilde{w}$, the weighted performance matrix $\tilde{V}$ is obtained as:

$$
\tilde{V}=\tilde{r} \otimes \tilde{w}
$$


Zhou et al. (1999) proposed a multi-objective fuzzy pattern recognition model to provide the global evaluation for every alternative with respect to all criteria. According to the maximum principle of membership degree, one can select the desired alternative from $n$ available alternatives. They defined the optimum membership degree of each alternative as:

$$
\mu_{j}=\frac{\left(d_{j}^{-}\right)^{2}}{\left(d_{j}^{*}\right)^{2}+\left(d_{j}^{-}\right)^{2}}
$$

In which $d_{j}^{*}$ and $d_{j}^{-}$are the synthetically weighted distance of alternative $j$ to the best and worst alternatives, respectively.

To calculate the distances of alternative $A_{j}$ to the ideal and anti-ideal alternatives, the fuzzy ideal weight distance is defined as Eqs. 14-15.

$$
\begin{aligned}
& d_{j}^{*}=\left[\sum_{i=1}^{m}\left(d\left(\tilde{r}_{i j}^{\tilde{w}}, \tilde{r}_{i}^{* w}\right)\right)^{q}\right]^{1 / q} \\
& d_{j}^{-}=\left[\sum_{i=1}^{m}\left(d\left(\tilde{r}_{i j}^{\tilde{w}}, \tilde{r}_{i}^{-w}\right)\right)^{q}\right]^{1 / q}
\end{aligned}
$$

Where $\widetilde{\sim}^{w}=\left[\tilde{r}_{i j}^{w}\right]$ is the weighted performance value of alternative $A_{j}$ with regard to criterion $C_{i}$ and $m$ is the number of criteria. In this study, we define the positive ideal solution $\tilde{r}_{i}^{*}=(1,1,1)$ and the anti-ideal solutions $\widetilde{r}_{i}=(0,0,0)$ under the criteria as references to measure alternatives' performances. For more information on different methods to measure the distance between fuzzy numbers refer to Hsieh and Chen (1999).

\section{Model Application to Karun System Development}

\subsection{Description of the Study Site}

In this study, the Karun system has been considered by different levels and different approaches. Karun is the largest river in Iran with average annual discharge of 14,690 MCM. Due to its high hydropower potential and large irrigation demand in Khuzestan plain, 12 reservoirs are either constructed, under construction, or being designed (Fig. 1). This study concentrates on Karun II(3), II(8), III, and IV from which Karun III reservoir is already constructed and is definitely a fixed member of all development and management options. For Karun II, two different alternatives are available at different sites, in which Karun $\mathrm{II}(8)$ is approximately $40 \mathrm{~m}$ shorter. Due to their pronounced environmental impacts, the Ministry of Energy has conducted an impact assessment study on all potential reservoirs emphasizing on Karun II(3), II(8), III, and IV as the largest ones. Table 2 presents the seven different development alternatives defined by the water authorities in Iran Water and Power Development Company (IWPDC) to assess the priority of any of the reservoirs and/or their combinations. All information on different alternatives is extracted from 


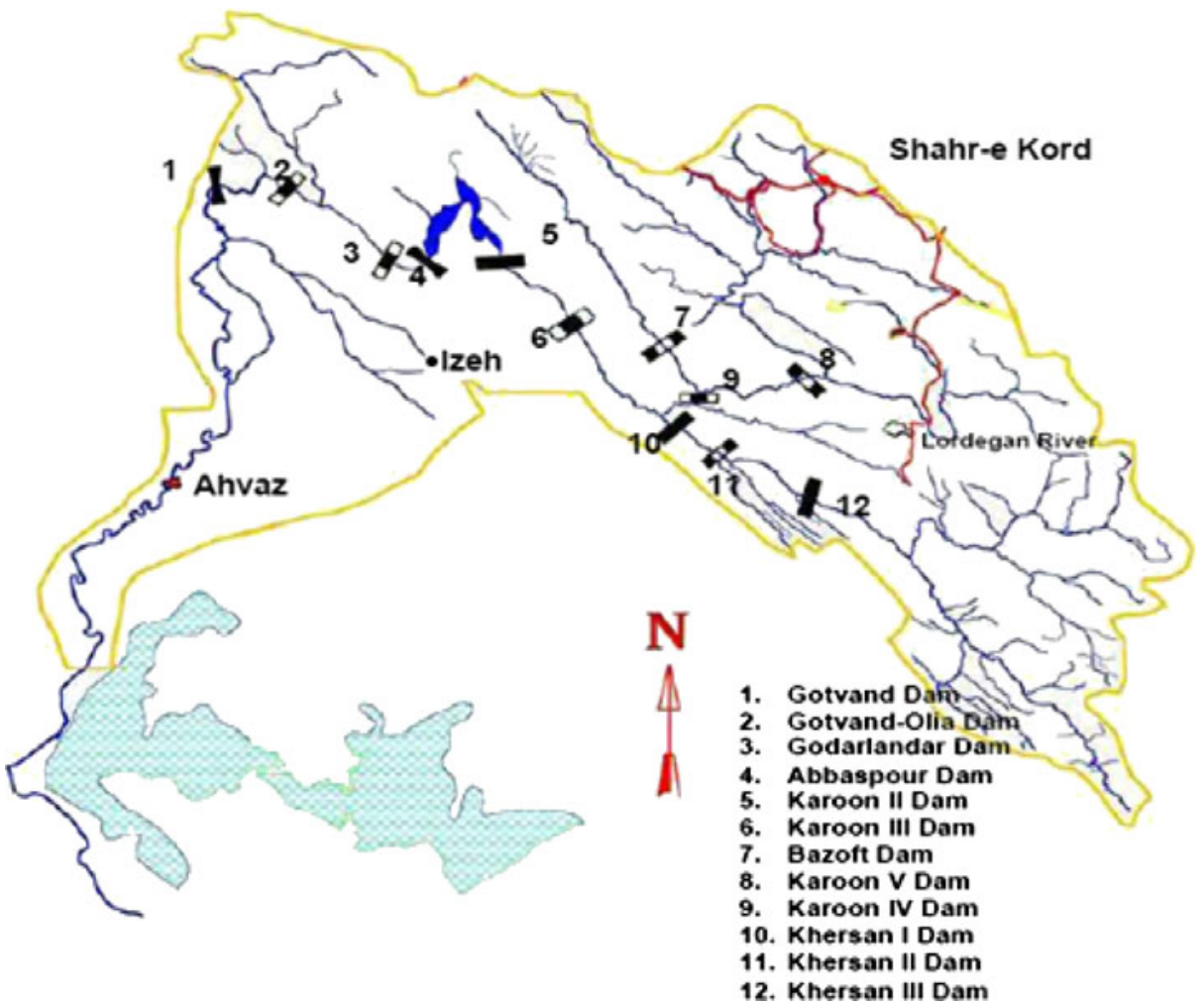

Fig. 1 Karun river basin development scheme

technical reports on individual reservoirs and is available on request from the first author (Hydroinformatic Research Center 2005).

Environmental impact assessment is generally complex in nature in that it involves a variety of qualitative factors arising from ecological, social, economical, and even political concerns. Because of imprecision in data, uncertainty in modeling and subjectivity in human judgment, decision making for environmental impact assessment (EIA) is actually a multi-criteria decision making problem under fuzzy environments.

\subsection{Modeling Approach}

This study employs a multi-level evaluation method for determination of the actual state of the water related environment from a joint ecological-socioeconomic

Table 2 Development alternatives in Karun river basin study

\begin{tabular}{ll}
\hline Option & Management option \\
\hline$A_{1}$ & Karun II(8), Karun III, Karun IV \\
$A_{2}$ & No development \\
$A_{3}$ & Karun II(3), Karun III, Karun IV \\
$A_{4}$ & Karun III, Karun IV \\
$A_{5}$ & Karun II(8), Karun III \\
$A_{6}$ & Karun III \\
$A_{7}$ & Current state (as is) \\
\hline
\end{tabular}


standpoint. The method is flexible since the number of basic indicators as well as third and second level criteria may be changed according to the characteristics of the system. This flexibility is reflected in the mathematical presentation of the methodology.

Clearly, there are systems which have few basic indicators and/or where no second level criteria is proposed. On the other hand, more than three level analysis and system decomposition may be needed if the water related environment is much more complicated. The specified three-level analysis seems a good compromise between sophistication and practical applicability.

In this study, a combination of the multi-attribute utility theory of United Nation Environmental Program (UNEP 1987) and the proposed fuzzy optimization method have been used to assess the alternatives proposed by the Karun river basin authorities as addressed in Table 2.

To implement the UNEP multi level evaluation method for alternative ranking in deterministic environment, the following step by step summary is given to evaluate the state of the water related environment (UNEP 1987).

1. Select the basic indicators (first level indicators)

2. Set up the best and worst values for all basic indicators

Table 3 First, second, and third level indicators and/or criteria

\begin{tabular}{|c|c|c|}
\hline Third level & Second level & First level \\
\hline \multirow[t]{15}{*}{$\overline{\text { Ecology }}$} & \multirow[t]{6}{*}{ Wild life } & Animal diversity \\
\hline & & Wood area \\
\hline & & Endanger species \\
\hline & & Local valuable species \\
\hline & & Fish diversity \\
\hline & & Pasture and irrigate lands \\
\hline & \multirow[t]{4}{*}{ Water quality } & Temperature and stratification \\
\hline & & TDS and sedimentation \\
\hline & & Eutrification \\
\hline & & River ecology \\
\hline & \multirow[t]{2}{*}{ Hydrology } & Stream flow \\
\hline & & Flooding (peak reduction) \\
\hline & \multirow[t]{3}{*}{ Geophysics } & Sites and views \\
\hline & & Erosion \\
\hline & & Induced seismicity \\
\hline \multirow[t]{15}{*}{ Socio-economic } & \multirow[t]{5}{*}{ Social issues } & Public participation \\
\hline & & Population resettlement \\
\hline & & Inundate houses \\
\hline & & Education \\
\hline & & Community spatial structure \\
\hline & \multirow[t]{5}{*}{ Economic } & Irrigation and animal husbandry \\
\hline & & Employment \\
\hline & & $\begin{array}{l}\text { Communication and } \\
\text { infrastructure }\end{array}$ \\
\hline & & Interaction with other projects \\
\hline & & Power generation $\left(\mathrm{gw}^{*} 10^{\wedge} 3\right)$ \\
\hline & \multirow[t]{2}{*}{ Sanitary } & Background pollution \\
\hline & & Public health \\
\hline & \multirow[t]{3}{*}{ Culture } & Tourism \\
\hline & & Local culture \\
\hline & & Historical site \\
\hline
\end{tabular}




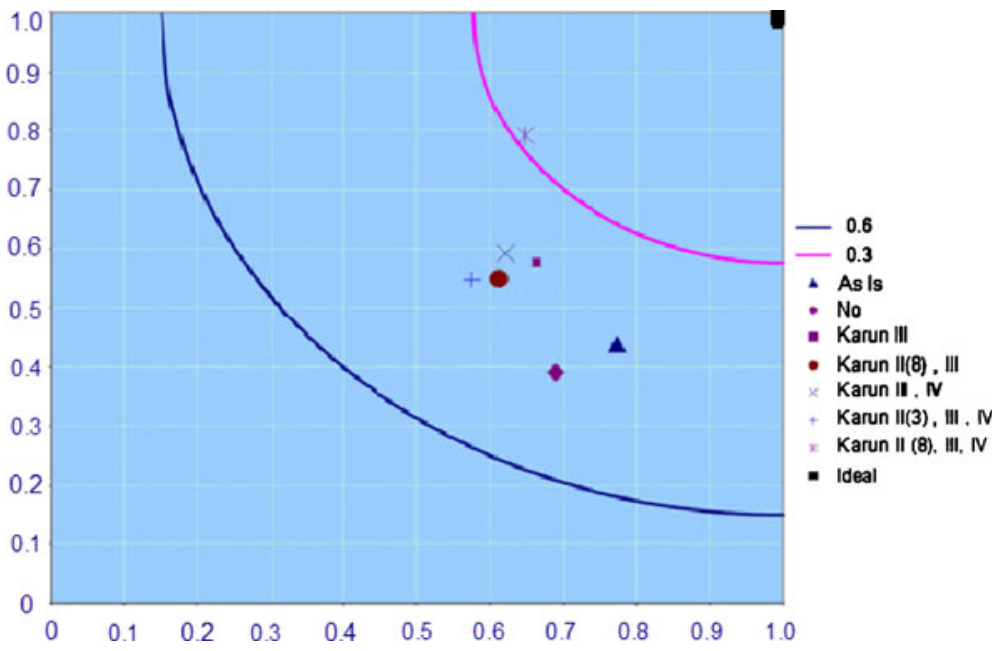

Fig. 2 Distance to ideal reference point based on UNEP deterministic approach

3. Estimate the value of indicator $i$ for the proposed option $j\left(x_{i j}\right)$

4. Normalize basic indicators and aggregate them to form second level criteria using the appropriate weights

5. Aggregate the second level criteria to obtain values for the third level criteria

6. The third level criteria (socio-economic and ecology) are then aggregated to obtain the distance measure from the ideal reference point.

7. Rank the options based on their distance measure from the ideal point.

The basic indicators, second, and third level criteria for both deterministic and fuzzy TOPSIS are listed in Table 3 . The results for the deterministic environment are presented in Fig. 2, where the first management option consisting $\mathrm{K}^{1} \mathrm{II}(8)$, KIII, and KIV receives the highest priority.

In the fuzzy UNEP technique, the importance weights of the criteria are obtained using pairwise comparison and are defined as linguistic variables of extremely important (EI), very very important (VVI), very important (VI), important (I), fairly important (FI), unimportant (U), and very unimportant (VU). Table 4 presents the ranges of fuzzy numbers assigned for different linguistic values of the criteria weights.

In the second stage of this research, the weights and/or attributes of the indicators and/or criteria at different levels are assumed to be fuzzy. To test the performance of the proposed fuzzy TOPSIS model in ranking the options, two different cases are examined. In the first case, linguistic weights for all assessment levels are presented by fuzzy numbers. Triangular fuzzy numbers for weights of indicators are defined based on the experts' opinions as outlined in Table 4. These fuzzy values are aggregated to obtain the distance from the reference level for alternatives' ranking. Results of the proposed fuzzy UNEP approach to evaluate the state of the system with second and third level fuzzy weights are presented in Fig. 3. As it can be seen,

${ }^{1}$ Karun 
Table 4 Linguistic values for evaluation of criteria weights

\begin{tabular}{ll}
\hline Linguistic values for criteria weights & Fuzzy number \\
\hline Extremely important (EI) & $(0.95,1,1)$ \\
Very very important (VVI) & $(0.7,0.85,1)$ \\
Very important (VI) & $(0.55,0.7,0.85)$ \\
Important (I) & $(0.35,0.5,0.65)$ \\
Fairly important (FI) & $(0.15,0.3,0.45)$ \\
Unimportant (U) & $(0,0.15,0.3)$ \\
Very unimportant (VU) & $(0,0,0.05)$ \\
\hline
\end{tabular}

the general ordering of the options remains the same as the deterministic TOPSIS approach. Results for the same case with the first, second, and third level fuzzy weights are given in Fig. 4. In the both cases, alternative $A_{1}$ receives the higher priority followed by alternatives $A_{6}, A_{4}$, and $A_{5}$.

Extending the fuzziness to the weight of the first level has slightly reduced the priority of alternative $A_{5}$ in comparison to alternatives $A_{4}$ and $A_{6}$ (Figs. 3 and 4). Also alternative $A_{3}$ takes the position of alternative $A_{7}$ and vice versa. Detailed results of the model for deterministic and fuzzy cases are available from the corresponding author upon request.

In the second case, the fuzzy performance measure of each decision option against each criterion is also considered. Considering the possibilistic nature of uncertainties in the information originating from the absence of sharp boundaries and linguistic presentation of the criteria weighs by the experts and the authorities, the criteria and their weights are considered as fuzzy numbers. Based on the deterministic results and experts' judgment, the fuzzy values for the subject criteria are obtained and used for the alternative's ranking. As an example, fuzzy performance measures and their associated fuzzy weights for the second level criteria are presented in Tables 5 and 6 , respectively. Results of the fuzzy UNEP approach to evaluate the state of the system with the second level fuzzy indicators are presented in Fig. 3. Results of the approach

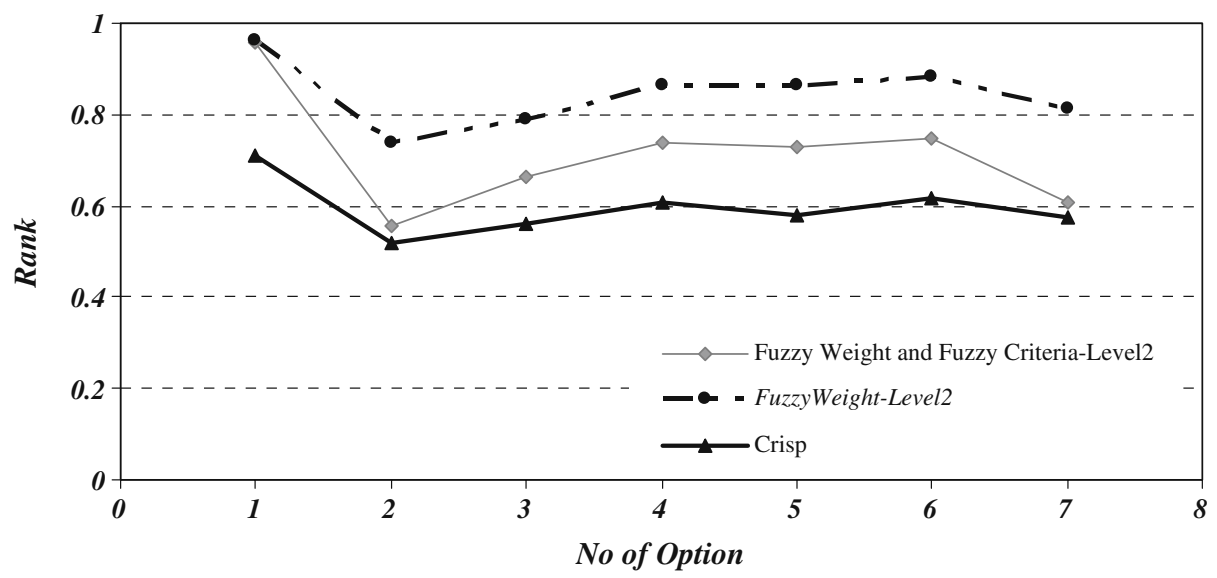

Fig. 3 Comparison of the overall ranking with UNEP and fuzzy UNEP approach (fuzzy nature of second level) 


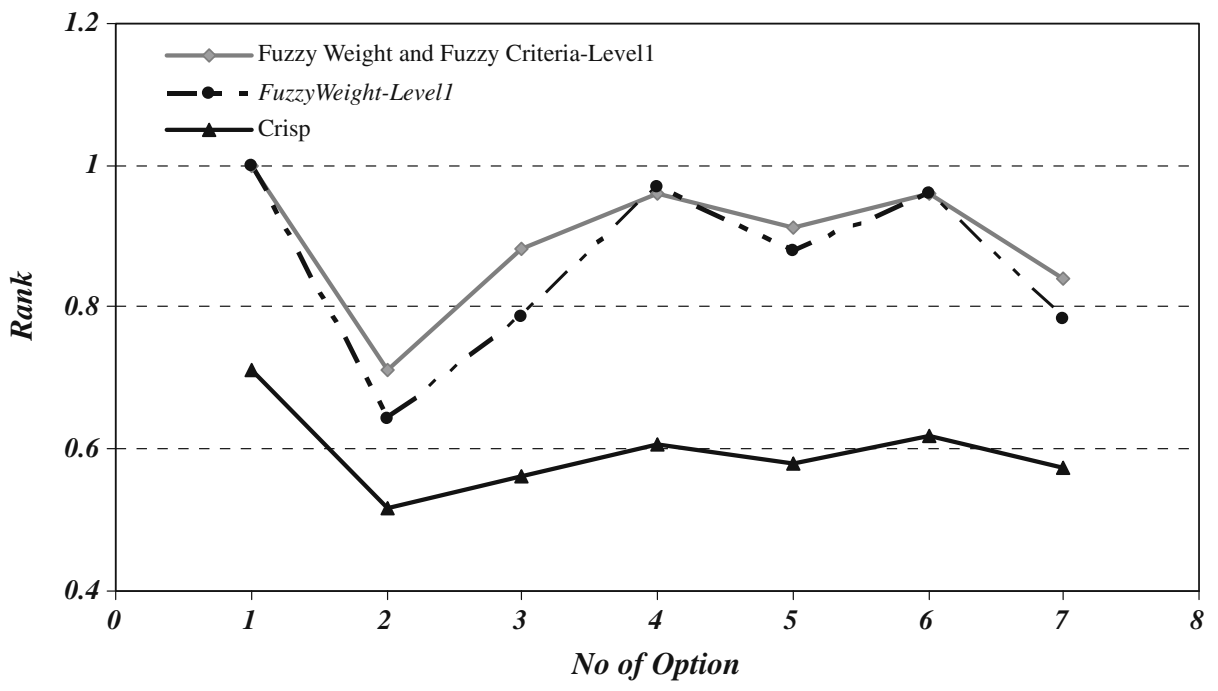

Fig. 4 Comparison of the overall ranking with UNEP and fuzzy UNEP approach (fuzzy nature of first level indicators)

show that with small margin alternative $A_{6}$ and $A_{4}$ are competitive alternatives after the $A_{1}$ alternative. Alternative $A_{1}$ with some operational measures to reducing the negative impacts on major criteria includes Karun II(8), Karun III, Karun IV reservoirs. As was expected, alternative $A_{1}$ has received the highest priority. In other words, $A_{1}$ is closest to the ideal alternative and farther from the anti-ideal alternative.

Extending the uncertainties to the first level, the weights and attributes of the first level indicators have also been considered as fuzzy numbers. Again, the positive and negative ideal solutions are determined, and the membership value of each alternative to the ideal solution (ranking order of each alternative) is calculated. The ranking measure is shown in the form of a rank index ranging from 0 to 1 . The larger the ranking index, the higher the priority of the option. The ranking order of each alternative in this case is presented in Fig. 4. In all cases the first alternative $A_{1}$ receives the highest priority, followed by the fourth and sixth alternatives. In this case, the ranking order is partly the same as the deterministic TOPSIS approach. Considering the fuzzy performance measure of each decision option against each criterion changes the priority for alternative $A_{3}$ with respect to $A_{7}$, as opposed to the deterministic TOPSIS.

\subsection{Sensitivity Analysis}

To test the sensitivity of the final ranks of the alternatives on the weights assigned to different criteria, a comprehensive sensitivity analysis has been carried out on the fuzzy weights of the first, second, and third level fuzzy indicators. Effects of assigning higher importance to the socio-economic criteria in the third level, compared to the ecology, was tested by increasing the socio-economic weight from important to veryvery important (I to VVI), while reducing that of ecology to fairly important (FI). 


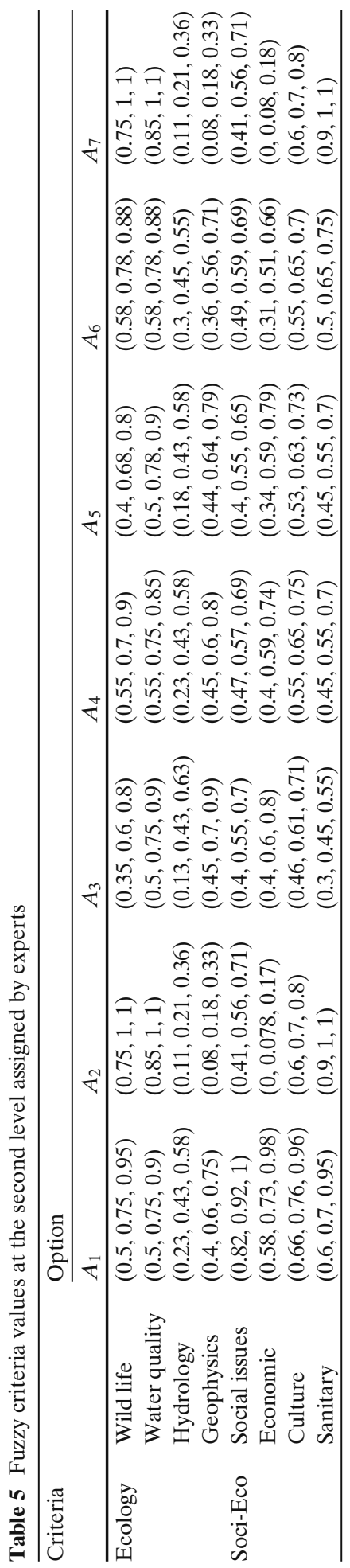


Table 6 Fuzzy weights for the second level criteria

\begin{tabular}{lll}
\hline Third level indicator & Second level indicator & Fuzzy weight \\
\hline Ecology & Wild life & $(0.55,0.7,0.85)$ \\
& Water quality & $(0.55,0.7,0.85)$ \\
& Hydrology & $(0.35,0.6,0.75)$ \\
Soci-Eco & Geophysics & $(0.35,0.5,0.75)$ \\
& Social issue & $(0.15,0.3,0.45)$ \\
& Economic & $(0.55,0.7,0.85)$ \\
& Culture & $(0.35,0.5,0.75)$ \\
& Sanitary & $(0.35,0.5,0.75)$ \\
\hline
\end{tabular}

This results in higher priority for $A_{4}$ alternative with respect to $A_{6}$, as opposed to the previous results. In another words, $A_{4}$ takes $A_{6}$ 's position in overall ranking (Fig. 5). It is important to note that priorities of other alternatives remain unchanged. In fact, alternatives $A_{4}$ and $A_{6}$ are more sensitive to that of socio-economic indicators.

On the other hand, if the importance of the ecology is raised by two levels (i.e., from I to VVI) and that of socio-economics is reduced from important to fairly important (i.e., I to FI) results in unchanging the ranks of alternatives (Fig. 5).

Sensitivity of the overall ranking to criteria weights in the first and second levels is carried out by systematically changing the proposed fuzzy weights. Two level increase in weights of socio-economic basic indicators (i.e., hydropower, employment, and infrastructures), while keeping those of other basic indicators constant, results in higher ranking for those alternatives with higher hydropower potentials. Explicitly, alternatives $A_{1}, A_{4}, A_{3}, A_{5}$, and $A_{6}$ receive higher priorities (Fig. 6). Again the first alternative receives the highest priority among all nominated alternatives.

Further sensitivity analysis on the weights of first and two level indicators reveals that alternatives ranking is not significantly sensitive to the weights of ecology and social criteria in the first level. In fact by modifying their weights from important to very important, the ranking remains unchanged (Fig. 6).

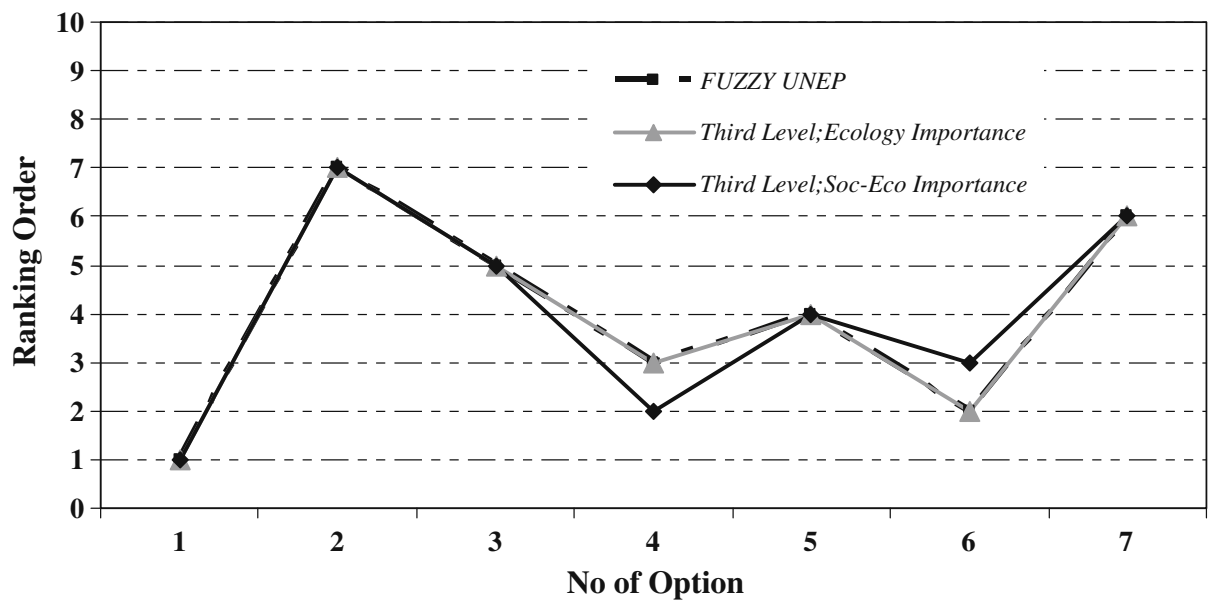

Fig. 5 Sensitivity analysis on the fuzzy weight of third level indicators 


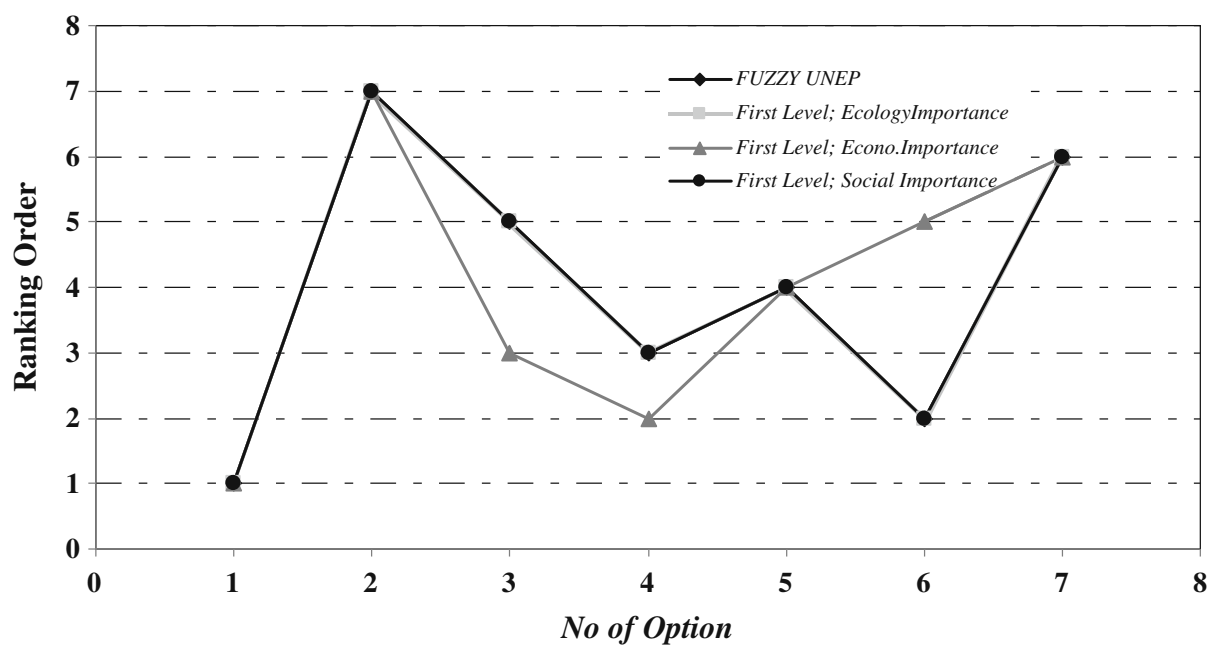

Fig. 6 Sensitivity analysis on the fuzzy weight of first level indicators

\section{Conclusion}

Optimal water projects ranking is a complex issue involving conflicting objectives (environmental, physical, socio-economic and health). There is a need for a reliable method to select and rank water development projects according to different objectives. MCDA provides an efficient means to develop future strategies and a system methodology to rank water projects in the presence of different objectives and constraints to satisfy the broad objectives defined by the sociopolitical conditions which are sometimes non-commensurable and conflicting. The proposed multicriteria decision making approach includes not only those factors within the water system itself but also a number of other socioeconomic, ecological, environmental, legislative, and political issues due to their direct or indirect relations to water management. The proposed MCDM model incorporates the uncertainties in both the quantification of the objectives and their importance in the ranking process. The model is easy to implement in a relatively large-scale water resource management and ranking problem. Both deterministic and fuzzy-based MCDM models are applied to rank projects in the Karun river basin of Iran. Results show that the ranking order remains more or less the same with both models. Both methods show that the option with the operational management scheme is the best option, followed by the 6th and 4th options with marginal differences.

Open Access This article is distributed under the terms of the Creative Commons Attribution Noncommercial License which permits any noncommercial use, distribution, and reproduction in any medium, provided the original author(s) and source are credited.

\section{References}

Abrishamchi A, Ebrahimian A, Tajrishy M, Mariño MA (2005) Case study: application of multicriteria decision making to urban water supply. J Water Resour Plan Manage 131(4):326-335 
Ashtian B, Haghighirad F, Makui A, Montazer GA (2009) Extension of fuzzy TOPSIS method based on interval-valued fuzzy sets. Applied Soft Computing 9:457-461

Barros MT-L, Tsai FT-C, Yang S-L, Lopes JEG, Yeh WW-G (2003) Optimization of large-scale hydropower system operations. J Water Resour Plan Manage 129(3):178-188

Borri D, Concilio G, Conte E (1998) A fuzzy approach for modeling knowledge in environmental systems evaluation. Comput Environ Urban Syst 22(3):299-313

Cai X, Lasdon L, Michelsen AM (2004) Group decision making in water resources planning using multiple objective analysis. J Water Resour Plan Manage 130(1):4-14

Cai YP, Huang GH, Lu HW et al (2009) I-VFRP: an interval-valued fuzzy robust programming approach for municipal waste management planning under uncertainty. Eng Optim 41(5): 399-418

Chen T-C (2000) Extensions of the TOPSIS for group decision-making under fuzzy environment. Fuzzy Sets Syst 114:1-9

Chu TC (2002) Selecting plant location via a fuzzy TOPSIS approach. Int J Adv Manuf Technol 20:859-864

Duckstein L, Treichel W, Magnouni S (1994) Ranking ground-water management alternatives by multicriterion analysis. J Water Resour Plan Manage 120:546-565

Ertuğrul I, Karakaşoğlu N (2008) Comparison of fuzzy AHP and fuzzy TOPSIS methods for facility location selection. Int J Adv Manuf Technol 39:783-795

Ertuğrul I, Güneş M (2007) Fuzzy multi-criteria decision making method for machine selection. Anal Des Intel Sys using SC Tech ASC 41:638-648, Springer, Heidelberg

Figueira J, Salvatore G, Ehrgott M (eds) (2005a) Multiple criteria decision analysis: state of the art surveys. Springer, Berlin

Figueira J, Mousseau V, Roy B (2005b) ELECTRE methods. In: Figueira J, Salvatore G, Ehrgott M (eds) Multiple criteria decision analysis: state of the art surveys. Springer, Berlin, pp 133-162

Flug M, Seitz HLH, Scott JF (2000) Multicriteria decision analysis applied to Glen Canyon Dam. J Water Resour Plan Manage 126(5):270-276

Fu G (2008) A fuzzy optimization method for multicriteria decision making: an application to reservoir flood control operation. Expert Syst Appl 34(1):145-149

Gershon L, Duckstein L, Aniff RM (1982) Multiobjective river basin planning with qualitative criteria. Water Resour Res 118(2):193-202

Goicoechea A, Hansen DR, Duckstein L (1982) Multi-objective decision analysis with engineering and business applications. Wiley, New York

Gonzalez B, Adenso-Diaz B, Gonzalez-Torre PL (2002) A fuzzy logic approach for the impact assessment in LCA. Resour Conserv Recycl 37:61-79

Hajkowicz S, Collins K (2007) A review of multiple criteria analysis for water resource planning and management. Water Resour Manage 21(9):1553-1566

Hsieh CH, Chen SH (1999) A model and algorithm of fuzzy product positioning. Inf Sci 121:61-82

Hyde KM, Maier HR, Colby CB (2004) Reliability-based approach to multicriteria decision analysis for water resources. J Water Resour Plan Manage 130(6):429-438

Hydroinformatic Research Center (2005) Karun reservoirs environmental impact assessment: final report. Iran University of Science and Technology, 4 volumes

Hwang CL, Yoon K (1981) Multiple attributes decision making methods and applications. Springer, Heidelberg

Kheireldin K, Fahmy H (2001) Multi-criteria approach for evaluating long term water strategies. Water Int 26(4):527-535

Lai E, Lundie S, Ashbol NJ (2008) Review of multicriteria decision aid for integrated sustainability assessment of urban water systems. Urban Water J 5(4):315-327

Larichev OI, Moshkovich HM (1995) ZAPROS-LM: a method and system for ordering multiattribute alternatives. Eur J Oper Res 82:503-521

Liu KFR, Huang SC, Liang HH (2009) A qualitative decision support for environmental impact assessment using fuzzy logic. J Environ Inform 13(2):93-103

Lund JR (1991) Random variables versus uncertain values: stochastic modeling and design. J Water Resour Plan Manage 117(2):179-194

Lund JR (2008) A risk analysis of risk analysis. J Contemporary Water Research \& Education 140:53-60

Morón B, Delgado Calvo-Flores M, Martín Ramos JM, Polo Almohano MP (2009) AIEIA: software for fuzzy environmental impact assessment. Expert Syst Appl 36:9135-9149 
Mourits M, Oude Lansink A (2006) Multi-criteria decision making to evaluate quarantine disease control strategies. In: Oude Lansink A (ed) New approaches to the economics of plant health. Springer, Heidelberg, pp 131-144

Netto OC, Parent E, Duckstein L (1996) Multicriterion design of long-term water supply in southern France. J Water Resour Plan Manage 122(6):403-413

Peche R, Rodríguez E (2009) Environmental impact assessment procedure: a new approach based on fuzzy logic. Environ Impact Asses Rev 29:275-283

Raju KS, Duckstein L, Arondel C (2000) Multicriterion analysis for sustainable water resources planning: a case study in Spain. Water Resour Manage 14(6):435-456

Saaty TL (2004) Decision making: the analytic hierarchy and network processes (AHP/ANP). J Syst Sci Syst Eng 13(1):1-35

Simonovic S (1989) Application of water resources systems concept to the formulation of a water master plan. Water Int 14:37-50

Tan Q, Huang GH, Cai YP (2009) A superiority-inferiority-based inexact fuzzy-stochastic programming approach for solid waste management under uncertainty. Environ Model Assess 15:381396. doi:10.1007/s10666-009-9214-6

Tecle A, Fogel M, Duckstein L (1988) Multi-criterion selection of wastewater management alternatives. J Water Resour Plan Manage 114(4):383-398

Triantphyllou E, Lin CT (1996) Development and evaluation of five fuzzy multi attribute decision making methods. Int J Approx Reason 14:281-310

UNEP (1987) Methodological guidelines for the integrated environmental evaluation of water resources development. International Hydrological Program, UNESCO, United Nations Environmental Program, Paris

Zhou HC, Wang GL, Yang Q (1999) A multi-objective fuzzy recognition model for assessing groundwater vulnerability based on the DRASTIC system. Hydrol Sci J 44:611-618

Zimmermann H-J (1996) Fuzzy set theory and its applications, 3rd edn. Kluwer, Boston 